\title{
BMJ Open Utility of social media and crowd- sourced data for pharmacovigilance: a scoping review protocol
}

\author{
Andrea C Tricco, ${ }^{1,2}$ Wasifa Zarin, ${ }^{1}$ Erin Lillie, ${ }^{1}$ Ba Pham, ${ }^{1}$ Sharon E Straus ${ }^{1,3}$
}

To cite: Tricco AC, Zarin W, Lillie $\mathrm{E}$, et al. Utility of social media and crowd-sourced data for pharmacovigilance: a scoping review protocol. BMJ Open 2017;7:e013474. doi:10.1136/bmjopen-2016013474

- Prepublication history and additional material is available. To view please visit the journal (http://dx.doi.org/ 10.1136/bmjopen-2016013474)

Received 13 July 2016 Revised 1 December 2016 Accepted 22 December 2016

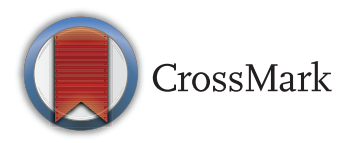

${ }^{1}$ Li Ka Shing Knowledge Institute of St. Michael's Hospital, Toronto, Ontario, Canada

2Epidemiology Division, Dalla Lana School of Public Health, University of Toronto, Toronto, Ontario, Canada ${ }^{3}$ Faculty of Medicine, Department of Geriatric Medicine, University of Toronto, Toronto, Ontario, Canada

Correspondence to Dr Andrea C Tricco; TriccoA@smh.ca

\section{ABSTRACT}

Introduction: Adverse events associated with medications are under-reported in postmarketing surveillance systems. A systematic review of published data from 37 studies worldwide (including Canada) found the median under-reporting rate of adverse events to be $94 \%$ in spontaneous reporting systems. This scoping review aims to assess the utility of social media and crowd-sourced data to detect and monitor adverse events related to health products including pharmaceuticals, medical devices, biologics and natural health products.

Methods and analysis: Our review conduct will follow the Joanna Briggs Institute scoping review methods manual. Literature searches were conducted in MEDLINE, EMBASE and the Cochrane Library from inception to 13 May 2016. Additional sources included searches of study registries, conference abstracts, dissertations, as well as websites of international regulatory authorities (eg, Food and Drug Administration (FDA), the WHO, European Medicines Agency). Search results will be supplemented by scanning the references of relevant reviews. We will include all publication types including published articles, editorials, websites and book sections that describe use of social media and crowd-sourced data for surveillance of adverse events associated with health products. Two reviewers will perform study selection and data abstraction independently, and discrepancies will be resolved through discussion. Data analysis will involve quantitative (eg, frequencies) and qualitative (eg, content analysis) methods.

Dissemination: The summary of results will be sent to Health Canada, who commissioned the review, and other relevant policymakers involved with the Drug Safety and Effectiveness Network. We will compile and circulate a 1-page policy brief and host a 1-day stakeholder meeting to discuss the implications, key messages and finalise the knowledge translation strategy. Findings from this review will ultimately inform the design and development of a data analytics platform for social media and crowd-sourced data for pharmacovigilance in Canada and internationally. Registration details: Our protocol was registered prospectively with the Open Science Framework (https://osf.io/kv9hu/).

\section{Strengths and limitations of this study}

- We will conduct a comprehensive literature search of multiple electronic databases and sources for difficult to locate and unpublished studies (or grey literature).

- Our scoping review will conform to the methodologically rigorous methods manual by the Joanna Briggs Institute.

- Numerous strategies will be used to disseminate our results widely.

- To increase the feasibility of our scoping review, we will limit to English and have one data abstractor and one verifier.

\section{INTRODUCTION}

Social media has gained unprecedented popularity worldwide. Currently, there are over 2.3 billion active social media users, and grows by an estimated 1 million new users every day. ${ }^{1}$ Social media platforms such as Twitter, Tumblr and Facebook are increasingly being used to discuss and share health issues. Statistics Canada revealed that over $80 \%$ of Canadians were internet users as of $2009,{ }^{2}$ and almost $70 \%$ of these individuals were using the internet to search for medical or health-related information. ${ }^{3}$ Social media and crowd-sourced data have been used to successfully extract information for surveillance of disease outbreaks, ${ }^{4}{ }^{5}$ health behaviour $^{6} 7$ and patient views on health issues. $^{8}$

The use of social media to exchange and discuss health information by the general public generates a large volume of unsolicited and real-time information. Health-related social networks, such as DailyStrength and MedHelp, attract users daily to discuss their health-related experiences, including use of prescription drugs, health products, side effects and treatments. During the 2004-2005 influenza season, social media listening by 
means of a Google 'click ad', which appeared on the search page when information seekers typed influenza-specific key words into the Google search engine, closely approximated the incidence of influenza cases. ${ }^{9}$ It was revealed that the Google ad click rate correlated more closely with retrospectively confirmed cases of influenza than the Physicians Sentinel Surveillance system for 'influenza-like illness'. ${ }^{9}$ Other researchers have also examined the use of social media for influenza outbreaks. ${ }^{10-12}$ Similarly, during the Canadian listeriosis outbreak, online search trends related to listeriosis correlated closely with laboratory-confirmed cases determined retrospectively, and preceded official announcements of an epidemic. ${ }^{13}$

Recently, researchers evaluated the types of information $^{14}$ including the prevalence of misinformation ${ }^{15}$ posted on Twitter and the Sina Weibo Chinese microblog platform related to the 2014-2015 Ebola epidemic. Given the observed predictive power of social media and crowd-sourced data as an information source for public health surveillance, a lot of interest has been generated about its use for surveillance of adverse events to health products, often referred to as pharmacovigilance.

Pharmacovigilance is defined as 'the science and activities relating to the detection, assessment, understanding and prevention of adverse effects or any other drug-related problem'. ${ }^{16}$ It includes drug safety surveillance activities to monitor incidents of adverse effects in real-life conditions. Adverse events, in particular to drug use, are a significant cause of morbidity and mortality, and are the fourth most common cause of death in hospitalised patients. ${ }^{17}$ Since many adverse events are not captured in randomised clinical trials, postmarketing surveillance of health and drug products is of paramount importance for drug and health technology industries and regulatory authorities, such as Health Canada, the US Food and Drug Administration (FDA) and European Medicines Agency (EMA). These governmental agencies require clinicians to report all suspected adverse events, but the voluntary nature of the reporting systems most likely contributes to the underreporting of adverse events. ${ }^{18-20}$ A systematic review of published data from 37 studies worldwide (including Canada) found the median under-reporting rate of adverse events to be $94 \%$ in spontaneous reporting systems. ${ }^{21}$ In response to the limitations in the current postmarketing surveillance systems, attention is being directed towards using social media and crowd-sourced data to detect adverse events and to improve consumer safety. Reviews have been conducted assessing social media for pharmacovigilance, such as a systematic review including 51 studies ${ }^{22}$ and a scoping review including 24 studies, ${ }^{23}$ but this is a rapidly evolving field and an updated scoping review with a comprehensive grey literature search may provide more clarity to the field. In addition, these previous reviews did not summarise pre-existing platforms that exist on this topic, which was requested by our knowledge user, Health Canada.
As such, we aim to assess the utility of social media and crowd-sourced data to monitor and detect adverse events related to health products. For the purpose of this review, health products include pharmaceuticals and drug products, medical devices, biologics, and natural health products. The specific research questions are:

1. What social listening and analytics platforms exist internationally to detect adverse events related to health products using social media and crowdsourced data? What are their capabilities and characteristics?

2. What is the validity and reliability of user-generated data from social media for surveillance of adverse events to health products?

\section{METHODS}

\section{Study design}

Our research objectives will be addressed using the scoping review methodology, which is a type of knowledge synthesis approach used to map the concepts underpinning a research area and the main sources and types of evidence available. ${ }^{24}$ This scoping review will be conducted in accordance with standard practices used by the Knowledge Synthesis Team within the Knowledge Translation Program of St Michael's Hospital. ${ }^{25}$ Our approach will be informed by the methodological framework proposed by Arksey and O'Malley, ${ }^{24}$ as well as the methodology manual published by the Joanna Briggs Institute for scoping reviews. ${ }^{26}$ This review has been commissioned by the Health Products and Food Branch (HPFB) of Health Canada and funded by the Canadian Institutes of Health Research Drug Safety and Effectiveness Network with a 6-month timeline.

\section{Protocol}

Our protocol was drafted using the Preferred Reporting Items for Systematic Reviews and Meta-analysis Protocols (PRISMA-P; see online supplementary appendix A), ${ }^{27}$ which was revised by the research team and members of Health Canada, and was disseminated through our programme's Twitter account (@KTCanada) and newsletter to solicit additional feedback. The final protocol was registered prospectively with the Open Science Framework on 6 September 2016 (https://osf.io/kv9hu/).

\section{Eligibility criteria}

The PICOS (Population, Intervention, Comparator, Outcome, Study design ${ }^{28}$ eligibility criteria are as follows:

\section{Population}

Patients of any age with an adverse event related to health products including pharmaceuticals and drug products, biologics, medical devices, and natural health products. ${ }^{29}$ Examples of pharmaceuticals and drug products include both prescription and non-prescription (over-the-counter) medicines, disinfectants and 
sanitisers with disinfectant claims. Biologics can include, but are not limited to: vaccines, insulin, serums, bloodderived products, hormones, growth factors and enzymes manufactured in bacterial, yeast or mammalian cell lines; and gene therapy and cell therapy products. Medical devices can include defibrillators, syringes, surgical lasers, hip implants, medical laboratory diagnostic instruments (including X-ray, ultrasound devices), contact lenses and condoms. Natural health products can include vitamins and minerals, herbal remedies, homoeopathic and traditional medicines, probiotics, and other products like amino acids and essential fatty acids. Adverse events, such as addiction and overdose from prescription medical products, are also eligible for inclusion. Adverse events related to programmes of care, health services, organisation of care, public health programmes, health promotion programmes and health education programmes will be excluded.

\section{Intervention}

Any data analytics or social listening platforms that enable the extraction of user-generated and crowdsourced data about adverse events to health products from social media are eligible for inclusion. Social media technology is defined as a web-based application that allows for the creation and exchange of usergenerated content. This includes, but is not limited to: websites, web pages, blogs, vlogs, social networks, internet forums, chat rooms, wikis and smartphone applications, where users have the ability to generate content (typically by providing posts and comments, often in an anonymous fashion or with limited identifying information) and are able to view/exchange content from and with others in an interactive digital environment. ${ }^{30}$ Crowd sourcing is the practice of obtaining needed services, ideas or content by soliciting contributions from a large group of people and especially from the online community rather than from traditional employees or suppliers. ${ }^{31}$ Social media listening and data analytics for public health surveillance related to non-communicable (eg, disease prevalence) and communicable diseases (eg, outbreak investigation) will be excluded.

\section{Comparators}

Any comparator is relevant for inclusion (eg, studies comparing one form of social media or crowd-sourced data to another or comparing social media with traditional reporting systems). In addition, studies without a comparator are eligible for inclusion.

\section{Outcomes}

There are two broad categories of outcomes that are of interest: (1) characteristics of social media listening and analytics platform (eg, data sources, scope of surveillance, capabilities, data extraction, preprocessing data, annotation, text mining methods, computational frameworks, added value to existing surveillance capacities, technical skills required, infrastructure support to implement and sustain, privacy and security of the data); and (2) validity and reliability of user-generated data captured through social media and crowd-sourcing networks (eg, relationship between medications and adverse events, algorithms or processes used to validate the data from social media, and related results of the evaluation).

\section{Study designs}

All types of publications including published articles, articles in conference proceedings, editorials, websites and chapters in textbooks are relevant.

\section{Time periods}

All periods of time and duration of follow-up are eligible.

\section{Other}

Given the 6-month timeline, only publications written in English will be considered for inclusion. If time allows, publications in other languages may be considered.

\section{Information sources and search strategy}

Comprehensive literature search strategies were developed by an experienced librarian for the following electronic bibliographic databases: MEDLINE, EMBASE and the Cochrane Library. The search strategy was peerreviewed by another expert librarian using the PRESS (Peer Review of Electronic Search Strategies) checklist. ${ }^{32}$ The final search strategy incorporated feedback from the peer review process and the complete search string for MEDLINE can be found in online supplementary appendix B. The full search terms for the other databases can be obtained by contacting the corresponding author. A trained library technician performed the final searches from inception to May 2016, exported the search results into Endnote and removed all duplicates.

A grey literature search was conducted according to the Canadian Agency for Drugs and Technologies in Health (CADTH) guide. ${ }^{33}$ Specifically, we searched 59 sources and websites of 119 relevant regulatory authorities for additional publications or pre-existing platforms of social media listening and data analytics. Examples of such social media listening and analytics platforms include the MedWatcher Social created in collaboration with the US FDA and Web-RADR (Recognising Adverse Drug Reactions) for the European Union regulators. ${ }^{34} 35$ See online supplementary appendix $\mathrm{C}$ for a full list of grey literature sources that were searched. Literature saturation will be ensured by searching the reference lists of relevant reviews. ${ }^{22} 2336$

\section{Study selection process}

To ensure high inter-rater reliability, a training exercise will be conducted prior to starting the screening process. Using our predefined eligibility criteria, a standardised questionnaire for study selection will be developed and tested on a random sample of 50 titles and 
abstracts (ie, level 1 screening) by all team members. The same training exercise will be repeated for screening of full-text articles (ie, level 2 screening). Subsequently, pairs of reviewers will screen citations and full-text articles for inclusion, independently, for level 1 and 2 screening. Inter-rater discrepancies will be resolved by discussion or a third adjudicator. All levels of screening will be conducted using Synthesi.SR, the proprietary online software developed by the Knowledge Synthesis Team. ${ }^{37}$

\section{Data items and data abstraction process}

We will abstract data on characteristics of the articles (eg, type of article or study, country of corresponding author), population characteristics (eg, type of patients, type of adverse events, disease condition), intervention characteristics (eg, type of social media or crowd-sourced data used) and outcomes (eg, data analytics/listening platform characteristics, data analytics used, validity and reliability of social media or crowd-sourced data). A standardised data abstraction form will be developed a priori and revised, as needed, after the completion of a training exercise.

Prior to data abstraction, we will complete a training exercise of the data abstraction form on a random sample of five articles. Subsequently, all included studies will be abstracted by pairs of reviewers, independently, with conflicts resolved by a third reviewer. If a large number of studies is identified $(>25)$, we will conduct data abstraction with one reviewer and one verifier.

\section{Risk of bias assessment or quality appraisal}

Since this is a scoping review aiming to map all available evidence, we will not conduct any risk of bias assessment or quality appraisal of included studies. This approach is consistent with the methods manual published by the Joanna Briggs Institute, ${ }^{26}$ as well as a database of scoping reviews on health-related topics. ${ }^{38}$

\section{Synthesis of results}

The synthesis will focus on providing a description of all social media listening platforms that exist internationally, and the validity and reliability of data from these social listening platforms, when available. This will be achieved by summarising the literature according to the types of participants, interventions, comparators and outcomes identified. Quantitative analysis will be conducted using descriptive statistics (eg, frequencies, measures of central tendency). In addition, we will consider qualitative analysis (eg, content analysis) for open-text data, as necessary. Two reviewers will conduct the initial categorisation coding independently, using NVivo software (NVivo V.10. Australia: International QSR, 2012), and the results will be discussed by the team. These reviewers will subsequently identify, code and chart relevant units of text from the articles using the categorisation code. Discrepancies will be resolved through team discussion.

\section{DISCUSSION}

\section{Implications}

Findings from this scoping review will inform decisionmakers of the types of social listening and analytics platforms that exist to extract user-generated data from social media for surveillance of adverse events to health products. This will inform Health Canada and other regulatory authorities internationally about the potential use of social media and crowd-sourced data for postmarketing surveillance.

\section{Dissemination}

The summary of results will be sent to Health Canada and other relevant policymakers and researchers working with the Drug Safety and Effectiveness Network in the form of a one-page policy brief. ${ }^{39}$ In addition, a 1-day stakeholder meeting (ie, consultation exercise) ${ }^{24}$ will be held to discuss the implications of our scoping review, key messages and to finalise the knowledge translation strategy. All relevant stakeholders will be invited to attend, as recommended by members from the Health Canada HPFB. This meeting will be essential to ensure extensive knowledge translation of our findings and to engage stakeholders and promote our research agenda. We will also present our results at an international conference and publish in an open-access journal. Finally, team members will use their networks to encourage broad dissemination of results.

Acknowledgements The authors thank Dr Elise Cogo for developing the literature search, Dr Jessie McGowan for peer-reviewing the literature search and Alissa Epworth for performing the database and grey literature searches and all library support, as well as Inthuja Selvaratnam and Theshani De Silva for formatting the manuscript.

Contributors ACT obtained funding, conceptualised the research and drafted the protocol. WZ helped write the protocol. EL and BP reviewed and edited the protocol. SES obtained funding, helped conceptualise the research and edited the protocol.

Funding This study has been funded by the Canadian Institutes of Health Research Drug Safety and Effectiveness Network. ACT is funded by a Tier 2 Canada Research Chair in Knowledge Synthesis. SES is funded by a Tier 1 Canada Research Chair in Knowledge Translation.

\section{Competing interests None declared.}

Provenance and peer review Not commissioned; externally peer reviewed.

Data sharing statement All data are available on request from the corresponding author.

Open Access This is an Open Access article distributed in accordance with the Creative Commons Attribution Non Commercial (CC BY-NC 4.0) license, which permits others to distribute, remix, adapt, build upon this work noncommercially, and license their derivative works on different terms, provided the original work is properly cited and the use is non-commercial. See: http:// creativecommons.org/licenses/by-nc/4.0/

\section{REFERENCES}

1. Smith K. Marketing: 96 amazing social media statistics and facts for 2016: Brandwatch Blog, 2016 [updated 7 Mar 2016]. https://www. brandwatch.com/2016/03/96-amazing-social-media-statistics-andfacts-for-2016/

2. Statistics Canada. Internet use by individuals, by selected characteristics 2010 [updated 10 May 2010]. http://www.statcan.gc. ca/tables-tableaux/sum-som/l01/cst01/comm35a-eng.htm 
3. Statistics Canada. Internet use by individuals, by type of activity (Internet users at home) 2010 [updated 10 May 2010. http://www. statcan.gc.ca/tables-tableaux/sum-som/101/cst01/comm29a-eng.htm

4. Twitter catches the flu: detecting influenza epidemics using Twitter. Proceedings of the conference on empirical methods in natural language processing. Association for Computational Linguistics. 2011.

5. Broniatowski DA, Paul MJ, Dredze M. National and local influenza surveillance through Twitter: an analysis of the 2012-2013 influenza epidemic. PLoS ONE 2013;8:e83672.

6. Jashinsky J, Burton $\mathrm{SH}$, Hanson $\mathrm{CL}$, et al. Tracking suicide risk factors through Twitter in the US. Crisis 2014;35:51-9.

7. Scanfeld D, Scanfeld V, Larson EL. Dissemination of health information through social networks: Twitter and antibiotics. Am $J$ Infect Control 2010;38:182-8.

8. Nakhasi A, Passarella R, Bell SG, Paul MJ, Dredze M, Pronovost P, editors. Malpractice and malcontent: Analyzing medical complaints in twitter. Association for the Advancement of Artificial Intelligence Fall Symposium Series; 2012.

9. Eysenbach G. Infodemiology and infoveillance: framework for an emerging set of public health informatics methods to analyze search, communication and publication behavior on the Internet. $J$ Med Internet Res 2009;11:e11.

10. Paul MJ, Dredze M, Broniatowski D. Twitter improves influenza forecasting. PLoS Curr 2014;6. doi:10.1371/currents.outbreaks. 90b9ed0f59bae4ccaa683a39865d9117

11. Santillana M, Nguyen AT, Dredze M, et al. Combining search, social media, and traditional data sources to improve influenza surveillance. PLoS Comput Biol 2015;11:e1004513.

12. Sharpe JD, Hopkins RS, Cook RL, et al. Evaluating Google, Twitter and Wikipedia as tools for influenza surveillance using Bayesian change point analysis: a comparative analysis. JMIR Public Health Surveill 2016;2:e161.

13. Chew C, Eysenbach G. Pandemics in the age of Twitter: conten analysis of Tweets during the $2009 \mathrm{H} 1 \mathrm{~N} 1$ outbreak. PLOS ONE 2010;5:e14118.

14. Fung IC, Hao Y, Cai J, et al. Chinese social media reaction to information about 42 notifiable infectious diseases. PLOS ONE 2015;10:e0126092.

15. Fung IC, Fu KW, Chan $\mathrm{CH}$, et al. Social media's initial reaction to information and misinformation on Ebola, August 2014: facts and rumors. Public Health Rep 2016;131:461-73.

16. World Health Organization. Essential medicines and health products: pharmacovigilance. 2016. http://www.who.int/medicines/areas/ quality_safety/safety_efficacy/pharmvigi/en/

17. Wester K, Jönsson AK, Spigset $\mathrm{O}$, et al. Incidence of fatal adverse drug reactions: a population based study. $\mathrm{Br} J$ Clin Pharmacol 2008;65:573-9.

18. van Der Hooft CS, Sturkenboom MC, van Grootheest K, et al. Adverse drug reaction-related hospitalisations: a nationwide study in The Netherlands. Drug Saf 2006;29:161-8.

19. Bates DW, Evans RS, Murff $\mathrm{H}$, et al. Detecting adverse events using information technology. J Am Med Inform Assoc 2003;10:115-28.

20. McClellan M. Drug safety reform at the FDA-pendulum swing or systematic improvement? N Engl J Med 2007;356:1700-2.
21. Mittmann N, Knowles SR, Gomez M, et al. Evaluation of the extent of under-reporting of serious adverse drug reactions: the case of toxic epidermal necrolysis. Drug Saf 2004;27:477-87.

22. Golder S, Norman G, Loke YK. Systematic review on the prevalence, frequency and comparative value of adverse events data in social media. Br J Clin Pharmacol 2015;80:878-88.

23. Lardon J, Abdellaoui $\mathrm{R}$, Bellet $\mathrm{F}$, et al. Adverse drug reaction identification and extraction in social media: a scoping review. J Med Internet Res 2015;17:e171.

24. Arksey H, O'Malley L. Scoping studies: towards a methodological framework. Int J Soc Res Methodol 2005;8:19-32.

25. Knowledge Translation Program. Canada: Knowledge Translation Program; 2016. http://knowledgetranslation.net/

26. Peters M, Godfrey C, Mclnerney P, Soares C, Hanan K, Parker D. The Joanna Briggs Institute Reviewers' Manual 2015: Methodology for JBI Scoping Reviews. 2015. Available from: http://joannabriggs. org/assets/docs/sumari/Reviewers-Manual_Methodology-for-JBIScoping-Reviews_2015_v2.pdf.

27. Shamseer L, Moher D, Clarke M, et al. Preferred reporting items for systematic review and meta-analysis protocols (PRISMA-P) 2015: elaboration and explanation. BMJ 2015;349:g7647.

28. Stone PW. Popping the (PICO) question in research and evidence-based practice. Appl Nurs Res 2002;15:197-8.

29. Health Canada. Drugs and Health Products. 2016 [updated 30 June 2016]. http://www.hc-sc.gc.ca/dhp-mps/index-eng.php

30. Naik P, Umrath T, van Stekelenborg J, et al. Regulatory definitions and good pharmacovigilance practices in social media: challenges and recommendations. Ther Innov Regul Sci 2015;49:840-51.

31. Merriam-Webster. Crowdsourcing. http://www.merriam-webster.com/ dictionary/crowdsourcing

32. McGowan J, Sampson M, Salzwedel DM, et al. PRESS Peer Review of Electronic Search Strategies: 2015 guideline statement. J Clin Epidemiol 2016;75:40-6.

33. CADTH. Grey matters: a practical tool for searching health-related grey literature: Canadian Agency for Drugs and Technologies in Health (CADTH). 2015. https://www.cadth.ca/resources/ finding-evidence/grey-matters

34. MedWatcher. MedWatcher for Enterprise 2016. https://medwatcher. org/about

35. WEB-RADR. WEB-RADR: Recognising Adverse Drug Reactions 2016. https://web-radr.eu/

36. Sinnenberg L, Buttenheim AM, Padrez K, et al. Twitter as a tool for health research: a systematic review. Am J Public Health 2017;107: e1-8.

37. Newton D. Synthesi.SR. Toronto, Canada: Knowledge Translation Program, St. Michael's Hospital, 2012.

38. Tricco AC, Lillie E, Zarin W, et al. A scoping review on the conduct and reporting of scoping reviews. BMC Med Res Methodol 2016;16:15.

39. Tricco AC, Soobiah C, Berliner S, Ho J, Ng C, Ashoor HS, SE. Efficacy and Safety of Cognitive Enhancers for Mild Cognitive Impairment: A Systematic Review and Meta-analysis; 2013. Available from: http://www.cihr-irsc.gc.ca/e/documents/dsen-abstracten.pdf 